\title{
LETTER
}

\section{Tb in a Global Health Exchange Program}

\author{
Matthew DeCamp, MD, $P h D^{7,2,9}$, John A. Crump, MB, ChB, DTM\&H $3,4,5,6$, Joce Rodriguez, \\ Gene Richardson, $M D^{8}$, Michele Barry, MD FACP ${ }^{7}$, and Jeremy Sugarman, $M D, M P H, M A^{1,2}$
}

'Johns Hopkins Berman Institute of Bioethics, Baltimore, MD, USA; ${ }^{2}$ Division of General Internal Medicine, Johns Hopkins School of Medicine, Baltimore, MD, USA; ${ }^{3}$ Division of Infectious Diseases and International Health, Department of Medicine, and Department of Pathology, Duke University Medical Center, Durham, NC, USA; ${ }^{4}$ Duke Global Health Institute, Duke University, Durham, NC, USA; ${ }^{5}$ Kilimanjaro Christian Medical Centre, Moshi, Tanzania; ${ }^{6}$ Kilimanjaro Christian Medical College, Tumaini University, Moshi, Tanzania; ${ }^{7}$ Center for Innovation in Global Health, Stanford University, Stanford, CA, USA; ${ }^{8}$ Department of Medicine, Stanford University School of Medicine, Stanford, CA, USA; ${ }^{9}$ Greenwall Fellow in Bioethics and Health Policy and Fellow in General Internal Medicine, Johns Hopkins University, Baltimore, MD, USA.

J Gen Intern Med 27(1):7

DOI: $10.1007 / \mathrm{s} 11606-011-1908-1$

(c) Society of General Internal Medicine 2011

To the Editor:- A series of publications in JGIM have begun to examine the personal health risks encountered in global health training programs, including tuberculin skin test (TST) conversion and blood and body fluid exposure ${ }^{1-3}$. Clearly, global health training programs abroad harbor risks for trainees. This can be due to disease prevalence (e.g., multidrug-resistant tuberculosis), the availability of personal protective equipment, and/or the extent to which trainees are prepared, among other factors.

Helping minimize risks to trainees in global health training programs is more than a logistic concern: it is an ethical obligation ${ }^{3-}$ 5. Merlin et al. $^{3}$ and Cooke ${ }^{4}$ engage in an important discussion about several ethical issues, including the ethical obligations of training programs to their trainees; the provision of adequate supervision; the importance of weighing of benefits and burdens to the host community; and the appropriate relationship between training programs and governing bodies where they operate.

The Working Group on Ethics Guidelines for Global Health Training ${ }^{5}$ recently published a set of ethics and best practice guidelines. These guidelines, developed through an international collaborative effort among multiple stakeholders, specifically reference the ethical obligations of sponsors, sending and host institutions, and trainees to develop and regularly update plans for helping ensure personal safety. The guidelines also address the importance of supervision; ensuring mutual and equitable benefits and costs among collaborators; demonstrating cultural competency; and meeting general professional and licensing standards.

Translating the guidelines into a practical and teachable format is the next challenge. To that end, we are currently developing an introductory Web-based ethics curriculum geared toward trainees who will engage in short-term $(<1$ year) training or service in global health. Using ten cases adapted from real-world scenarios, this brief curriculum uses video vignettes, multiple choice questions, and corrective feedback to address ten core ethical themes. Ensuring personal safety represents one theme. It will be freely available at http://www.ethicsandglobalhealth.org. Open and targeted recruitment strategies will evaluate the effectiveness of the curriculum in the near term.

Acknowledgments: The Wellcome Trust supported development of the WEIGHT guidelines; the Doris Duke Charitable Foundation supported the development of the web-based curriculum.

Corresponding Author: Matthew DeCamp, MD, PhD; Greenwall Fellow in Bioethics and Health Policy and Fellow in General Internal Medicine, Johns Hopkins University, 1809 Ashland Avenue, Baltimore, MD 21205, USA (e-mail: mdecamp1@jhmi.edu).

\section{REFERENCES}

1. Gardner A, Cohen A, Carter EJ. Tuberculosis among participants in an academic global health medical exchange program. J Gen Intern Med 2011; 26(8):841-5.

2. Dandu M. Trainee safety in global health. J Gen Intern Med 2011; 26 (8):826-7.

3. JS, Morrison G, Gluckman S, Lipschik G, Linkin DR, Lyon S, O'Grady E, Calvert H, Friedman H. Medical students in developing countries. J Gen Intern Med 2011; 26(8):833.

4. Cooke M. Medical students in developing countries: some benefits for sure but a mixture of risks. J Gen Intern Med 2011; 26(5):462-3.

5. Crump JA, Sugarman J. Working Group on Ethics Guidelines for Global Health Training (WEIGHT). Ethics and best practice guidelines for training experiences in global health. Am J Trop Med Hyg 2010; 83 (6):1178-82. 